\title{
Síndrome de Graham-Little-Piccardi-Lasseur: Relato de Um Caso
}

\author{
Monisa Martins Nóbrega', Thais Marques Feitosa Mendes Siqueira', Mariana Santiago Ormay', Daniel Lago Obadia2, \\ Alexandre Carlos Gripp ${ }^{3}$ \\ 'Médico Residente/Resident of Dermatology, Serviço de Dermatologia do Hospital Universitário Pedro Ernesto (HUPE/UERJ)- Rio de \\ Janeiro (RJ), Brasil \\ 2Pós-graduado em Dermatologia/Graduated in Dermatology, Universidade do Estado do Rio de Janeiro (UERJ); Professor substituto \\ de Dermatologia da Universidade do Estado do Rio de Janeiro (UERJ) - Rio de Janeiro (RJ), Brasil \\ ${ }^{3}$ Mestre em Dermatologia/Master in Dermatology. Professor assistente de Dermatologia/Professor of Dermatology; Responsável pela \\ enfermaria de Dermatologia/Head of the Dermatology Department, Hospital Universitário Pedro Ernesto - Universidade do Estado \\ do Rio de Janeiro (HUPE - UERJ) - Rio de Janeiro (RJ), Brasil.
}

RESUMO - A Síndrome de Graham-Little-Piccardi-Lasseur (SGLPL), variante folicular do Liquen Plano, caracteriza-se por apresentar, progressivamente: alopecia cicatricial multifocal do couro cabeludo associada a queratose folicular disseminada e hipotricose não cicatricial axilar e púbica. Os achados clínicos da síndrome podem estar presentes simultaneamente, embora a alopecia do couro cabeludo muitas vezes preceda os outros achados em meses a anos. Atinge principalmente mulheres na idade adulta, sendo considerada uma doença rara. A terapêutica desta dermatose é um desafio, apresentando resultados controversos. Relatamos o caso de uma paciente do sexo feminino, 75 anos, apresentando área de alopecia na região parietal direita, pápulas violáceas poligonais pruriginosas paravertebrais, máculas hipercrômicas com rendilhado branco na mucosa oral além de discreta diminuição dos pelos axilares. Após uso de hidroxizina associada à aplicação de propionato de clobetasol tópico e betametasona intralesional na placa alopécica observamos discreta melhoria.

PALAVRAS-CHAVE - Alopecia; Liquen Plano; Hipotricose; Síndrome.

\section{Graham-Little-Piccardi-Lasseur Syndrome: Report of a Case}

ABSTRACT - Graham-Little-Piccardi-Lasseur Syndrome (SGLPL) is characterized by a progressive multifocal scarring alopecia of the scalp associated with disseminated follicular hyperkeratosis and non-scarring axillary and pubic hypotrichosis. These clinical aspects may occur simultaneously, although alopecia of the scalp often precedes the other findings in months to years. It affects mainly adult females and is considered a rare disease. Treatment is challenging with many suggested medications but with controversial results. We report the case of a female patient with an area of alopecia in the right parietal region, paravertebral violaceous pruritic polygonal papules, hyperchromic macules with white reticular lesions on the oral mucosa as well as a slight hypotrichosis of the axillae. A slight improvement was observed after oral hidroxizine associated with topical clobetasol propionate and intralesional betamethasone in the limits of the alopecic area.

KEY-WORDS - Alopecia; Lichen Planus; Hypotrichosis; Syndrome.

\section{INTRODUÇÃO}

A síndrome de Graham-Little-Piccardi-Lasseur (SGLPL) é uma dermatose liquenoide rara, considerada uma forma de líquen plano folicular. Afecta principalmente mulheres na idade adulta e, clinicamente, apresenta-se pela tríade composta por alopecia cicatricial do couro cabeludo, alopecia não cicatricial das axilas e do púbis e erupção liquenoide folicular. Estes achados podem não ser encontrados simultaneamente. Geralmente, a SGLPL surge de forma esporádica e não tem caráter familiar e sua terapêutica é um desafio apresentando resultados controversos.
Correspondência: Thais Marques Feitosa Mendes Siqueira Travessa da Amizade, 120 apto 103 Vila da Penha

Rio de Janeiro- RJ Brasil CEP: 21221-370

E-mail: thathamfm@gmail.com
Recebido/Received

27 Junho/27 June 2016

Aceite/Accepted

10 Outubro/10 October 2016 


\section{Caso Clínico}

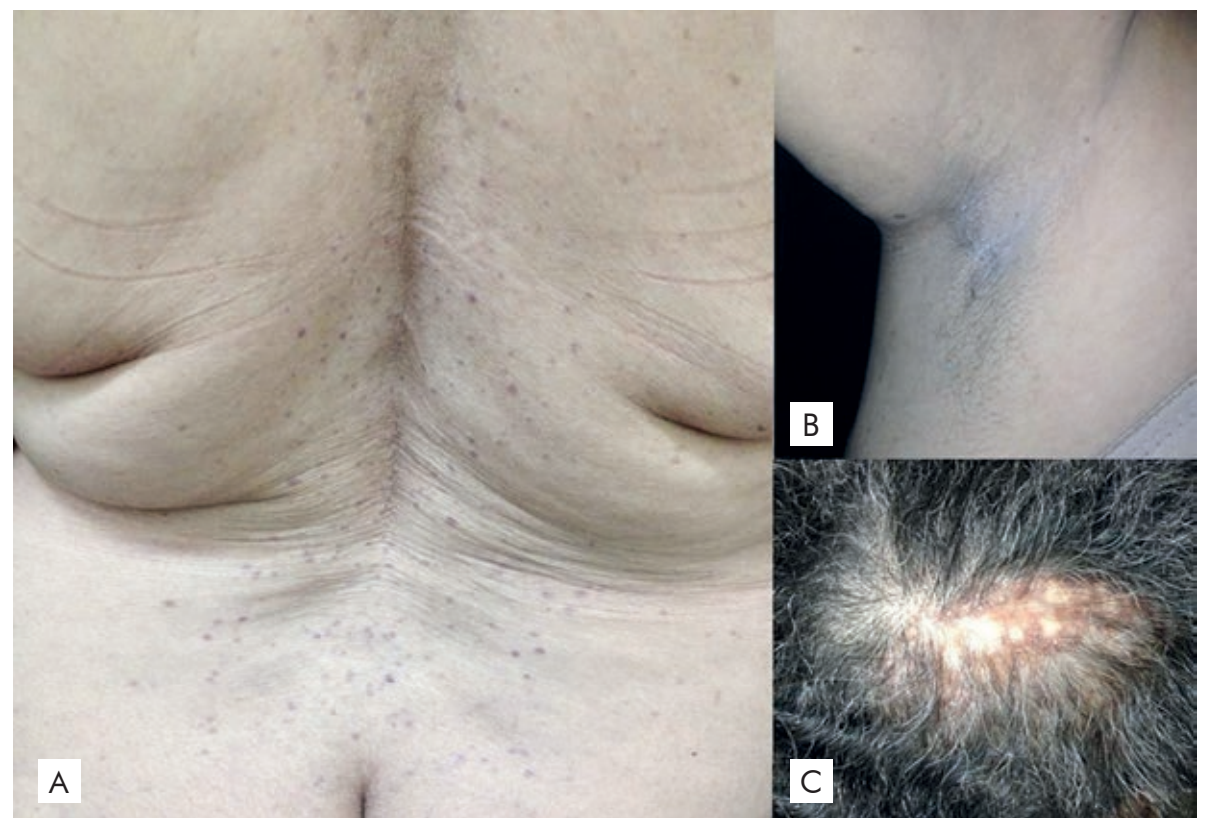

Figura 1 - (A) Pápulas violáceas, poligonais no dorso. (B) Hipotricose axilar com pelos finos e esparsos. (C) Placa alopécica com descamação perifolicular e áreas brancas atróficas.

\section{CASO CLÍNICO}

Paciente do sexo feminino, 75 anos, referindo queda de cabelo difusa e área de alopecia parietal direita com, aproximadamente, 6 meses de evolução. Relata ausência de resposta após uso de Minoxidil 5\% e D-pantenol $5 \%$ tópicos $1 x$ / dia por 2 meses. Refere também lesões pruriginosas paravertebrais tratadas sem sucesso com propionato de clobetasol tópico $2 x /$ dia.

Ao exame dermatológico observou-se placa de alopécia $\mathrm{com}$, aproximadamente $6 \mathrm{~cm}$ na região parietal direita associada a descamação perifolicular e regiões hipocrômicas e atróficas, associada a pápulas violáceas, poligonais, brilhantes, pruriginosas na região paravertebral máculas hipercrômicas com rendilhado branco na mucosa oral e, ainda, hipotricose discreta nas axilas (Fig 1), difícil de valorizar dada a idade da paciente. Ausência de alterações pilares na região púbica. Ao exame dermatoscópico no couro cabeludo observa-se descamação perifolicular tubular e eritema interfolicular (Fig. 2).

$\mathrm{Na}$ análise histopatológica de lesões da região dorsal e mucosa oral notou-se epiderme retificada com denso infiltrado inflamatório mononuclear liquenoide que agride a camada basal. No couro cabeludo observaram-se focos de infiltrado linfocitário liquenoide na epiderme e fibrose perifolicular (Fig 3).

Após o uso de hidroxizina associada a aplicação de propionato de clobetasol tópico e betametasona intralesional na placa alopécica observamos discreta melhoria com estabilização de seu tamanho e redução da descamação e eritema. As demais lesões permaneceram inalteradas e a paciente permanece em acompanhamento, sem extensão das lesões do couro cabeludo ao fim de mais 14 meses de seguimento.

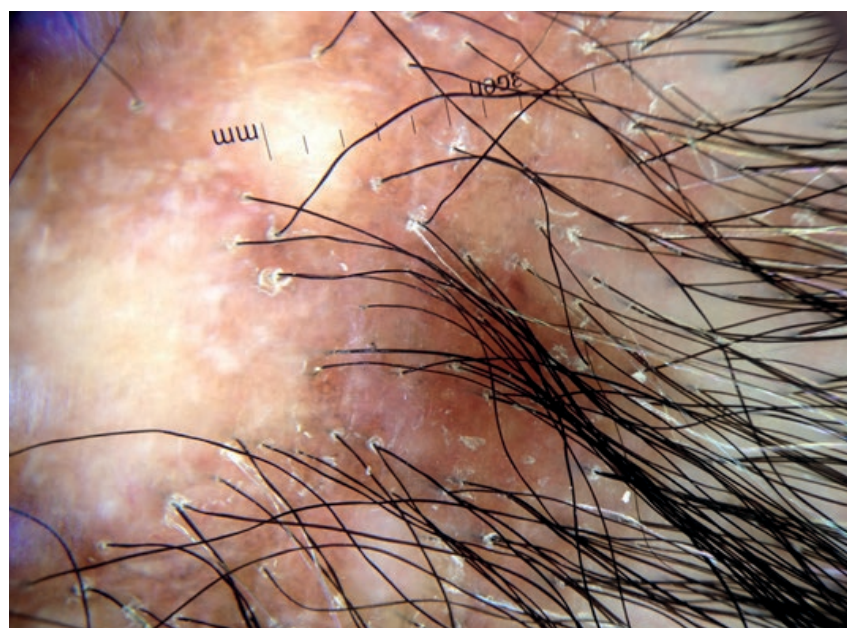

Figura 2 - Tricoscopia evidenciando descamação perifolicular tubular e eritema interfolicular.

\section{DISCUSSÃO}

Inicialmente descrita por Piccardi em 1913,' a Síndrome de Graham Little-Piccardi-Lassueur (SGLPL) é considerada uma patologia rara caracterizada por alopecia cicatricial no couro cabeludo, queratose espinulosa no tronco e extremidades, e alopecia não-cicatricial em axilas e púbis. ${ }^{2} \mathrm{~A}$ alopecia cicatricial, muitas vezes, precede qualquer erupção folicular por meses a anos. ${ }^{3}$ Os achados da tríade pertencente à SGLPL não precisam estar presentes simultaneamente. $O$ curso da doença é lentamente progressivo, pode levar meses ou anos, e é frequentemente crônica. ${ }^{3}$ É mais comum em 


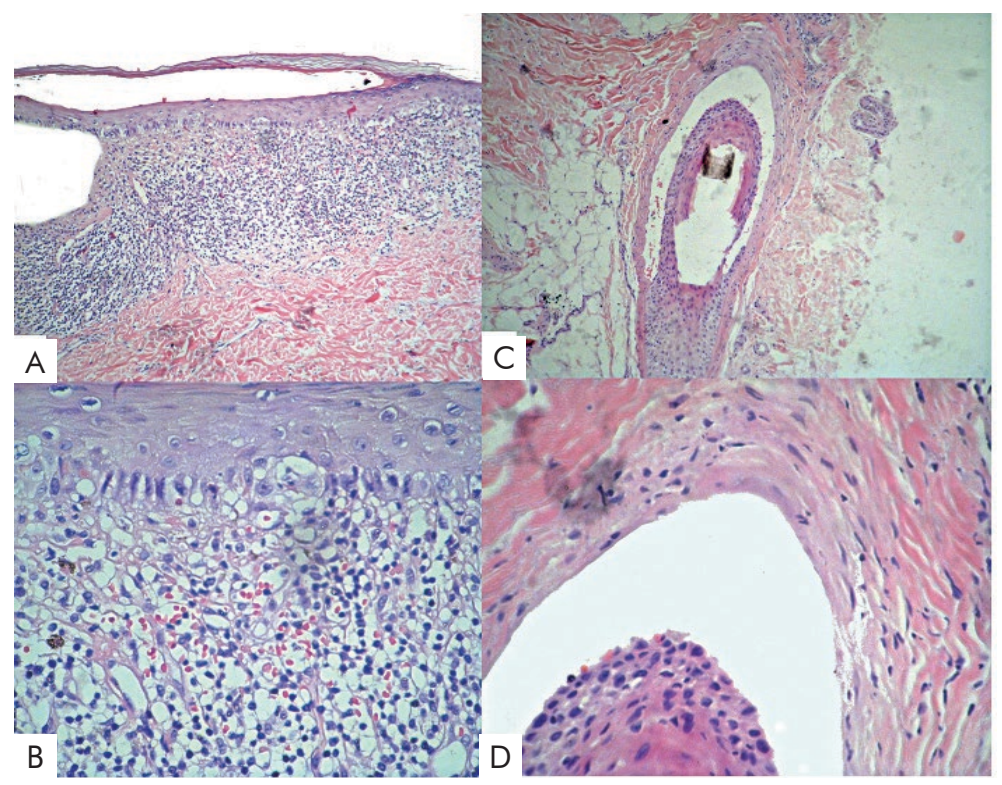

Figura 3 - Estudos histopatológicos de lesão dorsal (A e B) e do couro cabeludo (C e D) - (A) Epiderme retificada com denso infiltrado inflamatório em banda. (B) Detalhe do infiltrado inflamatório mononuclear liquenóide com agressão e degenerescência hidrópica da camada basal. (C) Focos de infiltrado linfocitário liquenóide na epiderme de um folículo piloso. (C) Fibrose perifolicular.

adultos entre 30 e 70 anos e é quatro vezes mais relatada em mulheres. ${ }^{4}$

A etiologia da SGLPL é desconhecida, no entanto, têm sido propostas várias hipóteses. A predisposição genética e associação imunológica com participação de antígenos HLA-DR tem sido sugerida, assim como associação ao vírus da hepatite $B(\mathrm{HBV})$ porque tanto a SGLPL quanto o líquen plano foram relatados após vacinação para HBV. ${ }^{3}$ Outras possíveis causas, como estresse neuropsicológico, níveis hormonais alterados e síndrome de insensibilidade androgênica, foram discutidas como possíveis causas ${ }^{3,5}$ porém nenhuma dessas causas foi encontrada na paciente em questão.

O exame dermatoscópico caracteriza-se pela presença de descamação perifolicular na porção proximal dos folículos pilosos com arranjo peculiar dos pontos cinza-azulados ao redor das unidades foliculares descrito como "padrão em alvo". Já a epiderme interfolicular apresenta-se com eritema na forma de vasos arboriformes. ${ }^{6}$

A histopatologia revela um infiltrado denso linfocítico perifolicular, com linfócitos estendendo-se para dentro da camada basal e alteração vacuolar focal da camada basal folicular. $^{3}$

O diagnóstico diferencial inclui outras causas de alopecia cicatricial, como pseudopelada de Brocq, lúpus eritematoso discoide, sarcoidose, mucinose folicular, foliculite e ceratose pilar decalvante atrofiante. ${ }^{7}$

O tratamento da SGLPL é difícil e tende a ter pouco êxito no que diz respeito tanto à alopecia cicatricial quanto à erupção de ceratose folicular. Na maioria dos pacientes, alopecia cicatricial do couro cabeludo não responde a intervenções médicas e resulta em perda de cabelo progressiva e permanente. Várias modalidades de tratamento são usadas na tentativa de retardar o processo, como por exemplo, corticosteroides tópicos, intralesionais e sistêmicos, retinoides, ciclosporina, fototerapia (PUVA), e antimaláricos. ${ }^{8}$ Os primeiros tiveram efeitos modestos na paciente descrita.

Conclui-se, portanto, que a SGLPL compreende um quadro generalizado de inflamação perifolicular sintomática que eventualmente evolui para destruição folicular irreversível. A melhor compreensão da sua etiopatogenia possivelmente possibilitará tratamentos mais eficazes no futuro.

Conflitos de interesse: Os autores declaram não possuir conflitos de interesse. Suporte financeiro: O presente trabalho não foi suportado por nenhum subsídio ou bolsa. Confidencialidade dos dados: Os autores declaram ter seguido os protocolos do seu centro de trabalho acerca da publicação dos dados de doentes. Protecção de pessoas e animais: Os autores declaram que os procedimentos seguidos estavam de acordo com os regulamentos estabelecidos pelos responsáveis da Comissão de Investigação Clínica e Ética e de acordo com a Declaração de Helsínquia da Associação Médica Mundial.

Conflicts of interest: The authors have no conflicts of interest to declare. Financing Support: This work has not received any contribution, grant or scholarship. Confidentiality of data: The authors declare that they have followed the protocols of their work center on the publication of data from patients. Protection of human and animal subjects: The authors declare that the procedures followed were in accordance with the regulations of the relevant clinical research ethics committee and with those of the Code of Ethics of the World Medical Association (Declaration of Helsinki). 


\section{Caso Clínico}

\section{REFERÊNCIAS}

1. Pai VV, Kikkeri NN, Sori T, Dinesh U. Graham-Little Piccardi Lassueur syndrome: an unusual variant of follicular lichen planus. Int J Trichology. $2011 ; 3: 28-30$.

2. Sperling LC. New look at scarring alopecia. Arch Dermatol. 2000; 136:235-42.

3. László FG. Graham-Little-Piccardi-Lasseur syndrome: case report and review of the syndrome in men. Int J of Dermatol. 2014; 53:1019-22.

4. James WD, Berger TG, Elston DM. Lichen planus and related conditions. In: Andrews' diseases of the skin. 9th ed. Philadelphia: WB Saunders Company; 2000. p.274-5.

5. Gutiérrez JV. Graham Little-Piccardi-Lassueur syndrome associated with androgen insensitivity syndrome (testicular feminization). J Eur Acad Dermatol Venereol. 2004; 18:463-6.

6. Pinto-Almeida T, Machado S, Selores M. Tricoscopia- estruturas tricoscópicas e sua aplicabilidade nas patologias do cabelo e do couro cabeludo. Rev Soc Port Dermatol Venereol. 2013;71:455-64.

7. Steglich RB, Tonoli RE, Pinto GM, Müller FM, Guarenti IM, Duvelius ES. Síndrome de Graham-Little Piccardi Lassueur: relato de caso. An Bras Dermatol. 2012;87:7757.

8. Sabia LM, Elias BL, Lessa PP, Ferreira FR, Lira ML, Mandelbaum SH. Síndrome de Graham- Little- Piccardi- Lassueur. Rev Soc Port Dermatol Venereol. 2014; 72:233-5. 